\title{
Abdominosacral Resection in the Management of Large- Size Retrorectal Tumors: A Report of 2 Rare Cases
}

\author{
Yuda Handaya ${ }^{1}$, Sutamto Wibowo ${ }^{2}$ \\ ${ }^{1}$ Digestive Surgery Division, Department of Surgery, Faculty of Medicine, Universitas Gadjah Mada/Dr. Sardjito Hospital, Yogyakarta; \\ ${ }^{2}$ Digestive Surgery Division, Department of Surgery, Faculty of Medicine, Airlangga University/Dr. Soetomo Hospital, Surabaya, Indonesia
}

Management of large-size retrorectal gastrointestinal stromal tumors (GISTs) is complex and challenging from diagnosis to treatment. This may create technical difficulties in surgical access and complete resection of the tumor. The abdominosacral resection has the benefit of improved visualization via the anterior incision, with enhanced exposure of the midrectal area, which makes resecting the tumor completely via the posterior approach easier. We report 2 cases of patients with a retrorectal GIST and neurofibromatosis type 1, one in a 27-year-old woman with a defecation complaint and the other in a 58-year-old woman with a defecation and urination complaint. Based on the anatomical pathology, both patients were diagnosed with a GIST. The tumors were excised via an abdominosacral resection. Retrorectal GISTs are rare, and abdominosacral resection allows complete resection of a large-size retrorectal GIST with low morbidity and an absence of functional impairment. The abdominosacral resection should be considered in certain situations.

Keywords: Gastrointestinal stromal tumors; Neurofibromatosis type 1; Rectal neoplasms; Disease management

\section{INTRODUCTION}

Gastrointestinal stromal tumors (GISTs) are the most common mesenchymal tumor of the gastrointestinal tract, but they are rare [1], with an estimated annual incidence of 10-20 cases/million population [2]. GISTs are derived from interstitial cells of Cajal, and they have three histological types: spindle, epithelioid, and mixed-type cells [3]. These tumors represent a malignant gastrointestinal tumor which occurs in approximately $5 \%-25 \%$ of patients with neurofibromatosis type 1 (NF1) [4]. GISTs are most common in the stomach (60\%) and small intestine (25\%), but they also arise in the colon, rectum, esophagus, mesentery, and omentum (15\% combined) [5]. Surgery is the first-line therapy

Received: March 24, 2017 • Accepted: December 1, 2017

Correspondence to: Yuda Handaya, M.D.

Digestive Surgery Division, Department of Surgery, Faculty of Medicine, Universitas Gadjah Mada/Dr. Sardjito Hospital, Jl. Kesehatan No. 1

Yogyakarta 55281, Indonesia

Tel: +62274581333, Fax: +62274581333

E-mail: yudahandaya@ugm.ac.id

ORCID code: https://orcid.org/0000-0003-0256-1955

(C) 2018 The Korean Society of Coloproctology

This is an open-access article distributed under the terms of the Creative Commons Attribution NonCommercial License (http://creativecommons.org/licenses/by-nc/4.0) which permits unrestricted noncommercial use, distribution, and reproduction in any medium, provided the original work is properly cited. for patients with primary resectable GISTs. The abdominosacral resection has the benefit of improved visualization via the anterior incision, with enhanced exposure of the midrectal area, which makes resecting the tumor completely via the posterior approach easier [6]. Herein, we report the rare case of a 27-year-old female patient with a retrorectal GIST and NF1, and a second case of a 58-year-old woman with a retrorectal GIST. Both patients were diagnosed with a large-size retrorectal GIST that was removed using the abdominosacral resection procedure.

\section{CASE REPORTS}

\section{Case 1}

This study has been approved by the Institutional Review Board of the Faculty of Medicine, Universitas Gadjah Mada Indonesia under permit ethic approval protocol number KE/0891/08/2018. The authors attest that full and informed consent was obtained from every single patient who have undergone medical treatment in our Hospital. The informed consent form also declared that patient data or samples will be used or educational or research purposes.

A 27-year-old female was admitted to the hospital with a defecation problem. The patient had a history of a Cesarean section and a laparotomy procedure because of a lower abdominal mass three years prior to admission. The patient also had had cutaneous neu- 

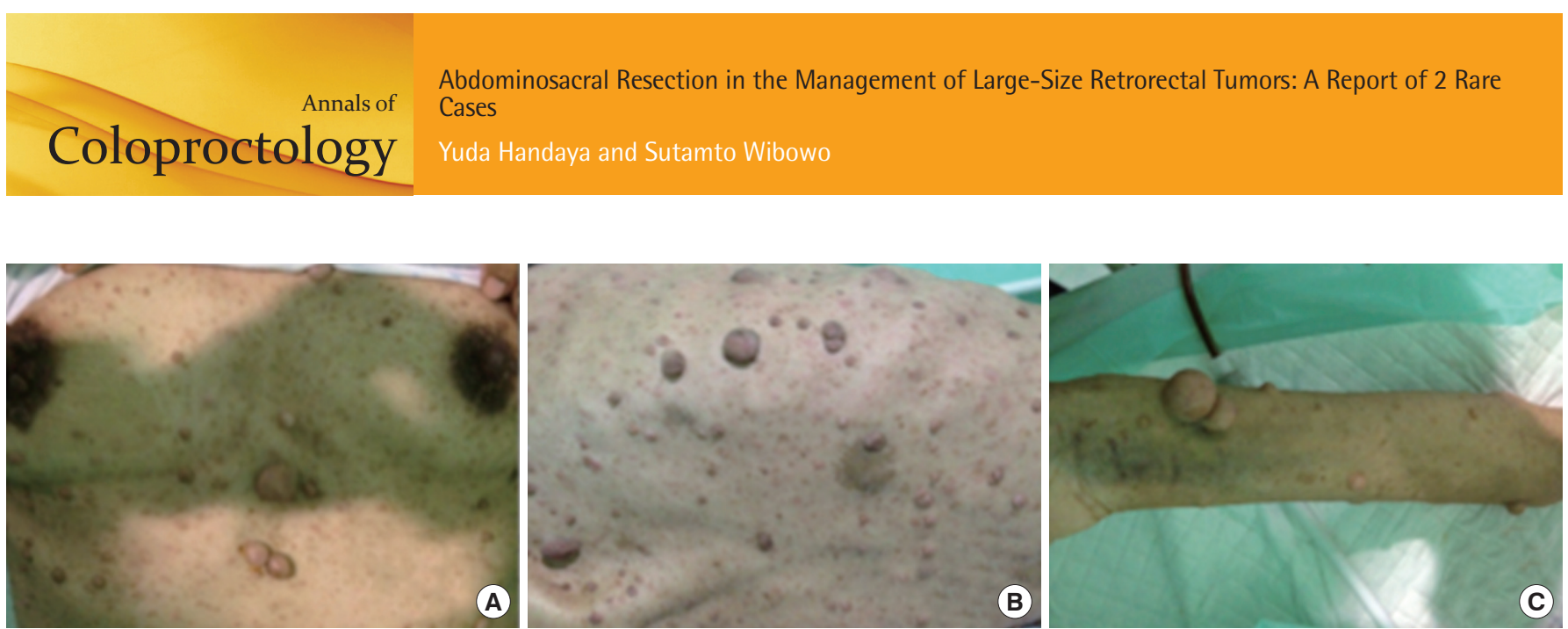

Fig. 1. Neurofibromas throughout the body of the patient: thorax and abdomen (A), back (B), and hand (C).
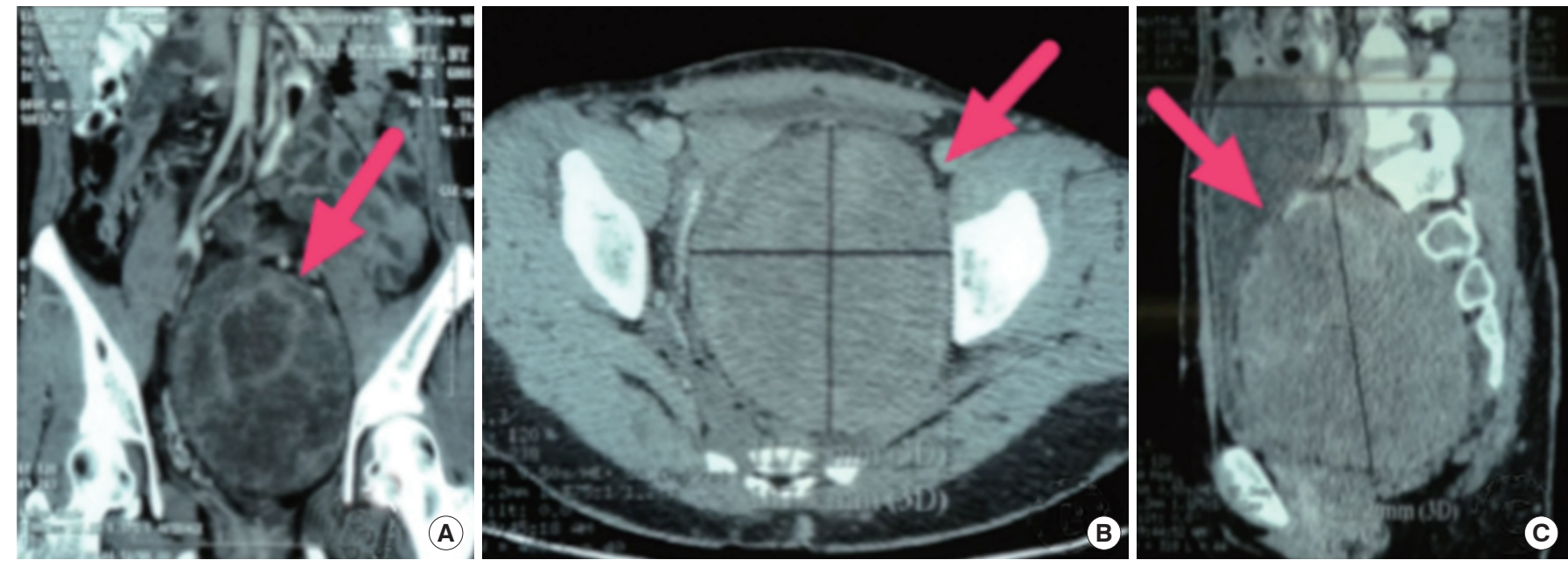

Fig. 2. Computed tomography scan views of the retrorectal tumor: coronal view (A), axial view (B), and sagittal view (C). The red arrows point toward the tumor mass.

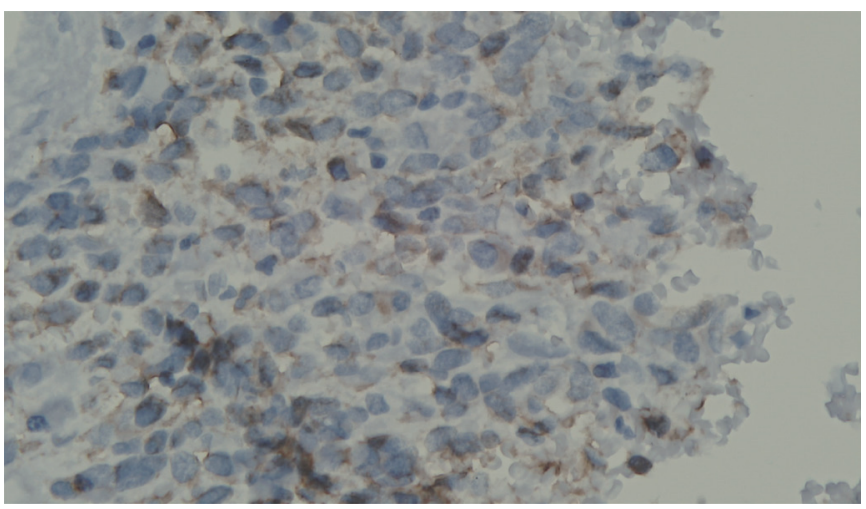

Fig. 3. Gastrointestinal stromal tumor sample viewed at $\times 200$ magnification indicating a negative result for CD117.

rofibromas throughout her body since she was 13 years old (Fig. 1).

Ultrasonography (USG) showed a $13 \times 12-\mathrm{cm}$ solid mass in the lower abdomen, which was displacing the urinary bladder and uterus anteriorly without direct invasion. Computed tomography (CT) showed a heterogeneous $10.73 \times 11.75 \times 11.23-\mathrm{cm}$ mass between the urinary bladder and the rectum, which was a suspected mesenchymal tumor (Fig. 2). We decided to perform a sigmoidostomy and biopsy. Anatomical pathology showed a benign soft tissue tumor with a differential diagnosis of a GIST and leiomyomas. However, immunocytochemical staining was negative for CD117, desmin, and smooth muscle actin (SMA) (Fig. 3).

We decided to perform a resection rather than use only an anterior approach so as to make exposure of the midrectum easy and to allow a complete excision. After the patient had been placed in a jack-knife position under general anesthesia, her buttocks were taped to the side of the table, and a paramedian skin incision was made. After a coccyx excision and sacrectomy (S3) had been performed, the tumor was identified and excised; it was attached to the distal segment of the sacrum (S3-S5) (Fig. 4A). A midline incision was made after the patient had been positioned in a supine position; the retrorectal tumor was then dissected intraabdomi- 

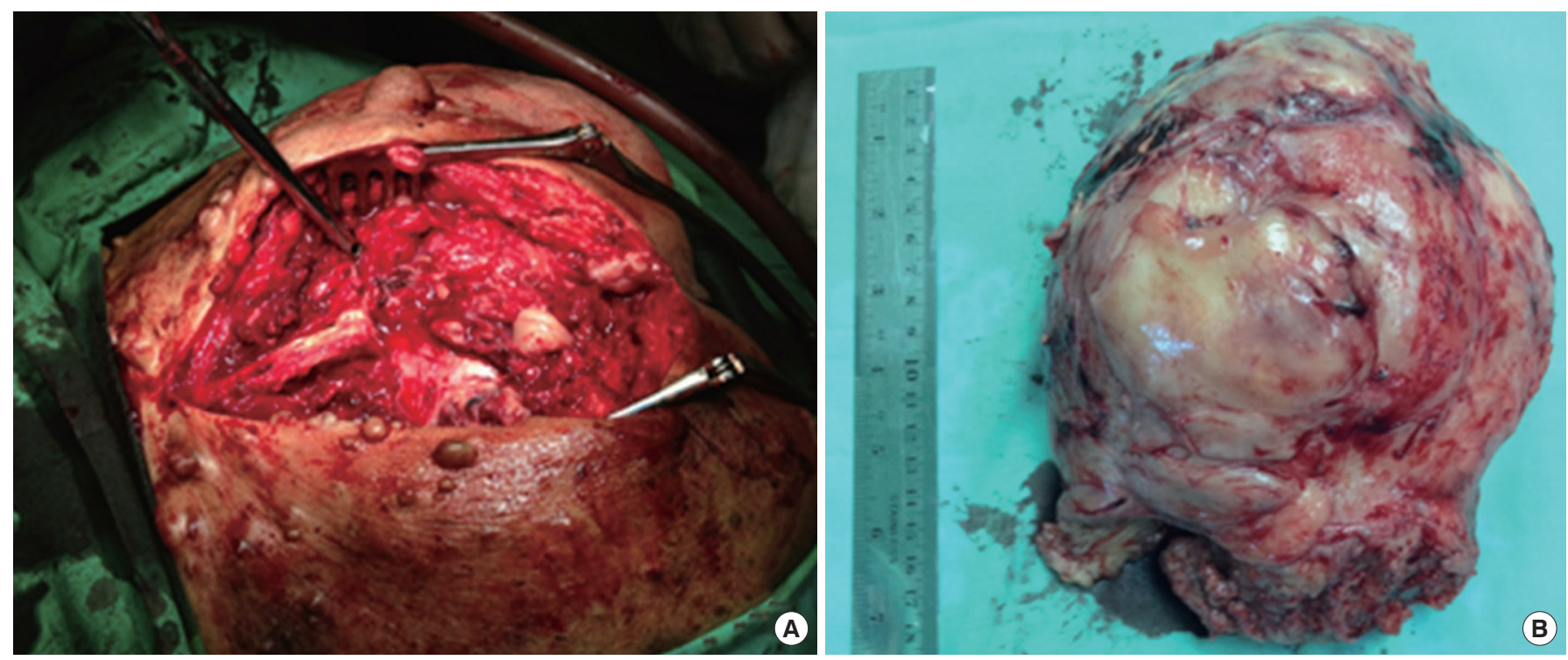

Fig. 4. (A) Posterior (Kraske) approach; sacrectomy S3-S5 segment. (B) The tumor mass was $10.73 \mathrm{~cm} \times 11.75 \mathrm{~cm} \times 11.23 \mathrm{~cm}$ in size and weighed $1.5 \mathrm{~kg}$.
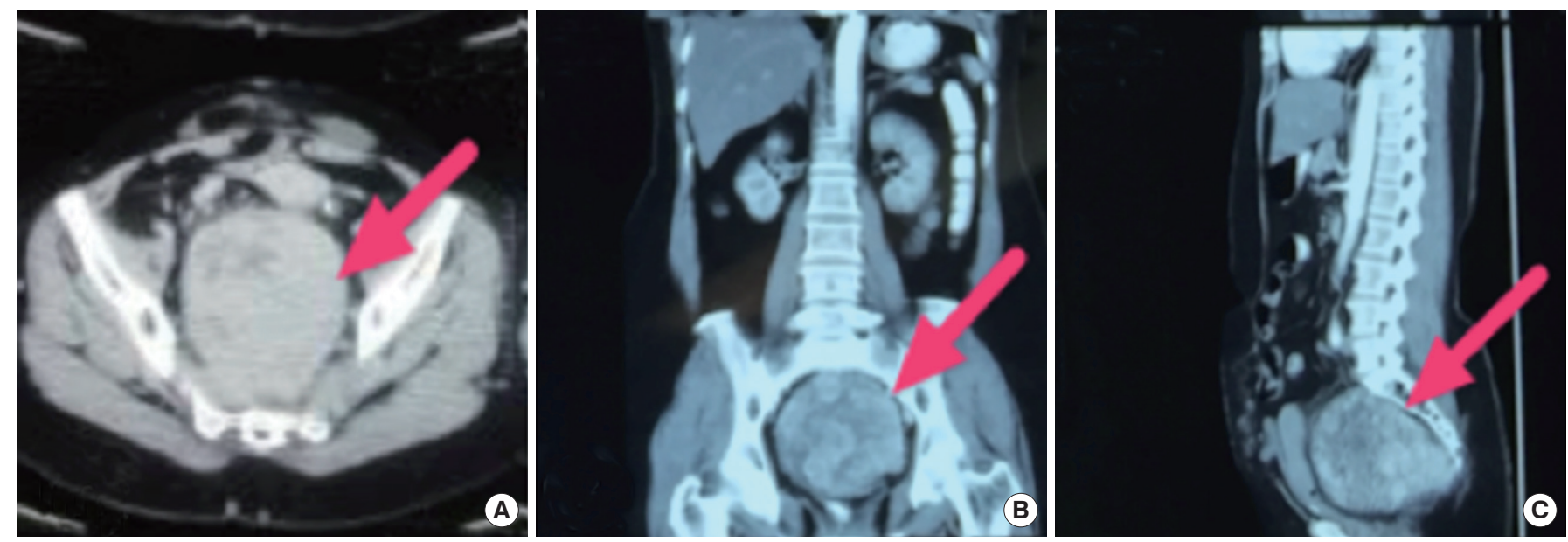

Fig. 5. Computed tomography scan view of the retrorectal tumor of the second patient: coronal view (A), axial view (B), and sagittal view (C). The red arrows point toward the tumor mass.

nally to release the attached cranial portion of the tumor, and it was separated from the surrounding organs (uterine, bladder, and left iliac vessels). Closure of the stoma (sigmoidostomy) was performed by using the end-to-end sewing technique after the tumor had been resected. The tumor was $10.73 \mathrm{~cm} \times 11.75 \mathrm{~cm} \times 11.23$ $\mathrm{cm}$ in size and weighed $1.5 \mathrm{~kg}$ (Fig. 4B). To control bleeding, we packed a continuous roll of gauze into the abdomen. The patient had an uneventful postoperative course and did not have fecal or urinary incontinence. The patient was administered $400 \mathrm{mg}$ of Imatinib daily as an adjuvant treatment for the GIST. No evidence of recurrence was seen at 1 year after surgery.
Case 2

A 58-year-old woman was referred to our hospital with a history of a bilateral salpingo-oophorectomy. Upon abdominal examination, we found tenderness and a suprapubic mass. Bimanual examination revealed that her cervix was at a fixed forward position and that a palpable solid mass was present on the posterior of the vagina. An extraluminal mass was found on digital rectal examination. Abdominal USG showed a solid, round mass with increasing peritumoral vascularization in the right adnexal region. The mass pushed the uterus in the left anterior-lateral direction. An abdominal CT scan showed an inhomogeneous $15 \times 15 \times 17$ $\mathrm{cm}$ mass on the anterior of the sacrum, which pushed on the rec- 

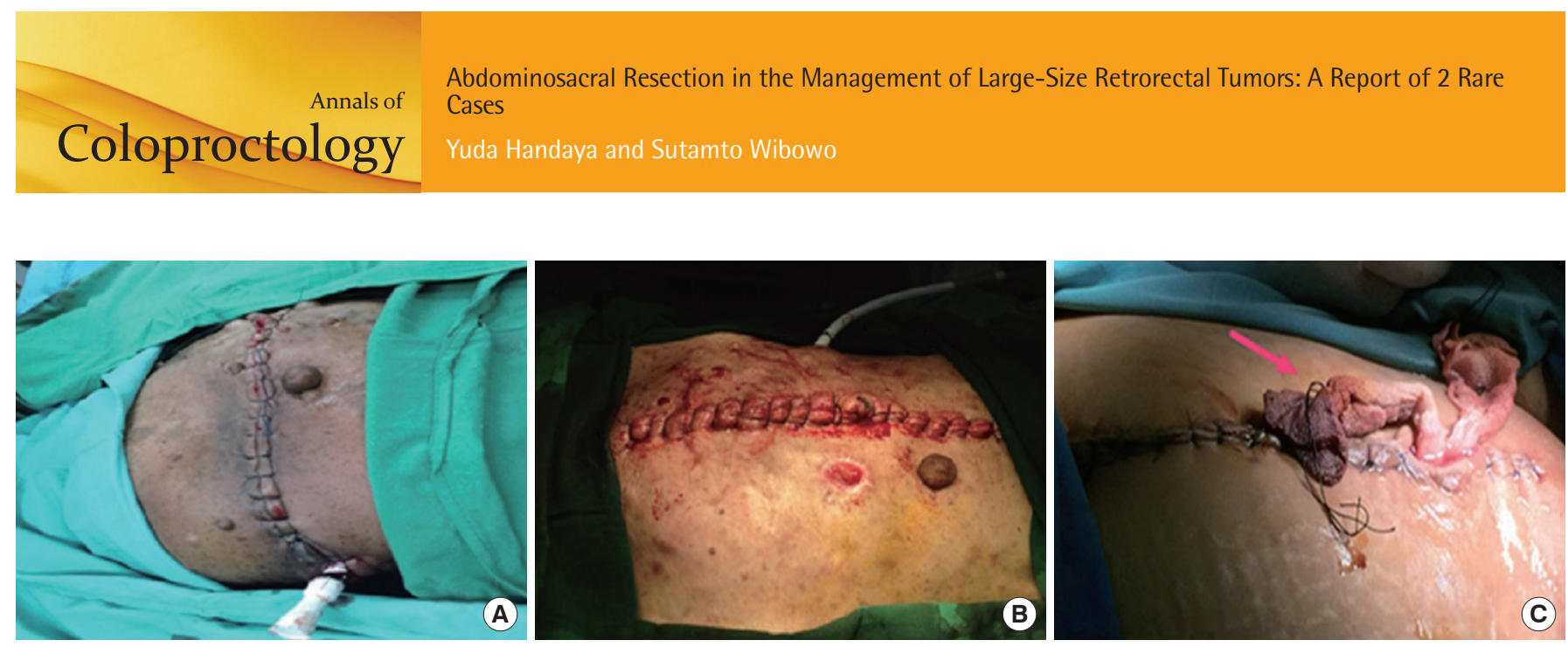

Fig. 6. Posterior (Kraske) approach (A), abdominal approach (B), and continuous roll gauze (C).

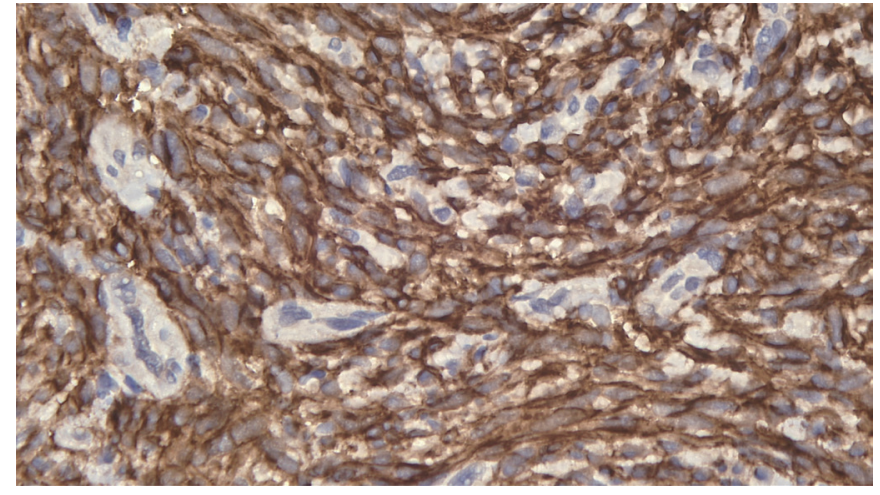

Fig. 7. Gastrointestinal stromal tumors samples viewed at $\times 200$ magnification with brown staining indicating a positive result for CD117.

tum and uterus (Fig. 5).

We performed an abdominosacral resection to remove the tumor (Fig. 6A, B). The blood loss was estimated to be up to 2,000 $\mathrm{mL}$, and resuscitation fluids, including blood and colloids in a ratio of 1:1, were used to prevent hypovolemic shock. To control the bleeding, we packed a continuous roll of gauze in the abdomen (Fig. 6C). The anatomical pathology results showed a GIST, and immunocytochemical staining was positive for CD117 (Fig. 7). The patient was administered $400 \mathrm{mg}$ of Imatinib daily as an adjuvant treatment for the GIST. The patient had a satisfactory recovery after surgery, with no fecal or urinary incontinence at one month after surgery.

\section{Operative procedure}

The step-by-step operative procedure is as follows:

1. Under general anesthesia, the patient was intubated.

2. The patient was positioned prone in the jack-knife position, we plan to resect inferior part (lower of imaginar lines at S23) of tumor with sacral resection technique and Superior part (upper imaginar lines S2-3) of tumor with transabdominal (Fig. 8).

3. Collaboration was done with the orthopedic surgeon for sacrectomy (S2-3) to the coxygeous region and the urology

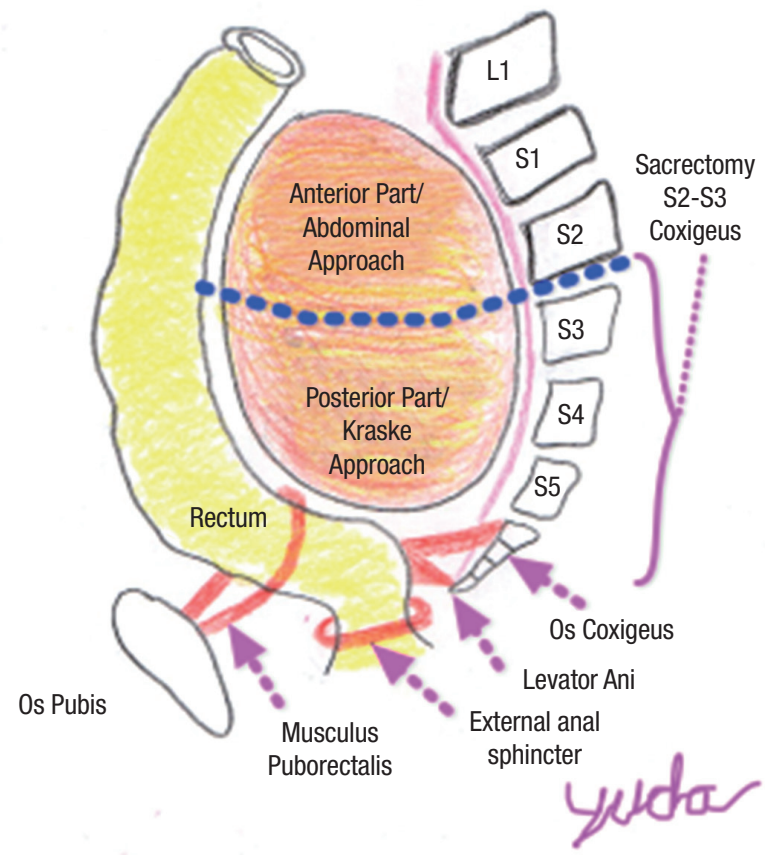

Fig. 8. Schematic drawing of the abdominosacral resection technique.

surgeon for double J stent insertion.

4. A midline longitudinal skin incision was made from the lumbosacral junction to the coccyx.

5. The sacral fascia was exposed from the S2 level to the tip of the coccyx.

6. Tissue dissection was performed inferiorly to expose the tip of the coccyx and then came around anterior to it.

7. The anococcygeal ligament was transected at a distance from the anal sphincter and, working ventral to the coccyx, the levator ani muscles were detached from it and retracted laterally to allow an approach to the presacral space.

8. A coccyx excision and sacrectomy (S3) was performed with a Kerrison rongeur.

9. The tumor was identified, and it was attached to the distal 
segment of the sacrum (S3-5).

10. A finger dissection was used to mobilize the rectum, creating a plane between the posterior aspect of the rectum and the ventral part of the sacrum including the anterior surface of the tumor.

11. Waldeyer's fascia was dissected to create an avascular plane between the mesorectal fascia propria and the presacral fascia.

12. An abdominal midline incision was made after the patient had been placed in a supine position.

13. The retrorectal tumor was then dissected intraabdominally to release the attached cranial portion of the tumor, and it was separated from the surrounding organs (uterine, bladder, and left iliac vessels).

14. An en bloc excision of the tumor was done with wide tumorfree margins.

15. To control the profuse bleeding, we packed three continuous rolls of gauze smeared with Vaseline and chloramphenicol ointment into retrorectal space to prevent infection and strong adhesion. Each continuous roll gauze was $2 \mathrm{~m}$ in length.

\section{DISCUSSION}

The GIST is the most common mesenchymal tumor of the gastrointestinal tract in the general population [1], with an estimated annual incidence of 10-20 cases/million population [2]. Across most ages, the incidence of GIST is similar in men and women [7]. Adult GISTs are rarely seen in patients younger than 40 years of age [8], but are prevalent in patients 50-70 years of age [7]. In our first case, the patient was a relatively young, 27-year-old female. GISTs are most commonly present in the stomach $(60 \%)$ and small intestine (25\%), but they also arise in the colon, rectum, esophagus, mesentery, and omentum (15\% combined) [5]. Based on our experience, this patient's case is rare because the GIST was located in the sacrum retrorectally. Furthermore, a tumor with a large size, as seen in this patient, is rare.

On the basis of the anatomical pathology results, both of our patients were diagnosed as having a GIST. Immunohistochemical staining was CD117-negative and CD117-positive in the first and the second patient, respectively. A study by Rubin et al. [9] suggested that approximately $10 \%$ of GISTs are c-kit negative. CD117 expression can be found in over 95\% of GISTs. However, CD117 expression is not found in the $5 \%$ of GISTs that are c-kit negative. Some authors believe that a few GISTs may not express CD117 [10]. We believe that our first case can be categorized as a CD117negative GIST. Based on the immunoreactivity to S-100 and SMA/desmin, CD 117-negative tumors can be divided into 4 groups. The current concept is that GISTs are derived from stem cells that differentiate into interstitial cells of Cajal, which are positive for CD117 on immunohistochemistry. Therefore, GISTs without CD117 expression are probably better regarded as a 'null phenotypic' when immunostaining for SMA/S-100 is also negative, as opposed to the 'prototypic' GISTs that are immunopositive for CD117 [11]. GIST samples from the second patient, when viewed at $\times 200$ magnification, exhibited brown staining, which is indicative of a positive result for CD117.

The management of a GIST of the sacrum is complex and challenging from diagnosis to treatment. This is because of its rarity and heterogeneous clinical scenarios and because the surgical procedures that are generally used are extensive and aggressive, aiming for complete tumor resection and a potential cure [12]. The posterior approach is reserved for small lesions below the level of S3. However, when the tumor size is large, the posterior approach is not sufficient. Therefore, an abdominosacral resection aimed to access the boundary of the posterior and caudal portion of the tumor through a posterior approach is required, which is enhanced using an abdominal approach to access or mobilize the anterior and the cephalic portions of the tumor. In this manner, the tumor can be removed completely. This approach to the management of a GIST is similar to that reported by Maddah et al. [13], who achieved a wide margin in sacral tumors that were often large and that required a combined posterior and anterior resection or an abdominosacral approach. The combined approach has the benefits of improved visualization via the anterior incision and enhanced exposure of nerve roots afforded by the posterior approach [6]. For tumors that span the S3 vertebral body, a combined abdominoperineal approach is often required, especially when sacral or pelvic sidewall invasion is present.

The potential occurrence of bleeding during surgery has been previously reported, especially in cases of a large-sized tumor. Anatomically, the presacral venous plexus runs into the pelvic fascia covering the anterior aspect of the sacrum. It is formed by the 2 lateral sacral veins, the middle sacral vein, and the veins communicating between them. These veins are avalvular and communicate via the basivertebral veins with the internal vertebral venous system [14]. The reported incidence of presacral hemorrhage ranges from $4.6 \%$ to $9.4 \%$ [15]. This bleeding can be life threatening and difficult to control, and intra-abdominal packing may be a life-saving method for controlling presacral hemorrhage. Pelvic packing with iodine gauze has been the most commonly used. To control the bleeding, we used continuous roll gauze packing in the abdomen. Unlike packed gauze placed in the abdomen, which requires a relaparotomy procedure to remove the gauze, the use of continuous roll gauze pads does not require a relaparotomy; the pads are removed by pulling out the ends of the gauze after having moistened them with normal saline solution. In our experience, the bleeding can be stopped by using this method.

In conclusion, a retrorectal GIST is not rare nowadays, but large tumors such as the ones seen in these cases are rare. Surgical treatment of patients with a retrorectal GIST is challenging because of the complex regional anatomy. An abdominosacral resection approach allows complete resection of a large-size retro- 
rectal GIST while achieving low morbidity and avoiding functional impairment. An abdominosacral resection should be considered in certain situations.

\section{CONFLICT OF INTEREST}

The authors declare that they have no conflicts of interest.

\section{REFERENCES}

1. Duffaud F, Blay JY. Gastrointestinal stromal tumors: biology and treatment. Oncology 2003;65:187-97.

2. Maeyama H, Hidaka E, Ota H, Minami S, Kajiyama M, Kuraishi A, et al. Familial gastrointestinal stromal tumor with hyperpigmentation: association with a germline mutation of the c-kit gene. Gastroenterology 2001;120:210-5.

3. Hirota S, Isozaki K, Moriyama Y, Hashimoto K, Nishida T, Ishiguro S, et al. Gain-of-function mutations of c-kit in human gastrointestinal stromal tumors. Science 1998;279:577-80.

4. Yantiss RK, Rosenberg AE, Sarran L, Besmer P, Antonescu CR. Multiple gastrointestinal stromal tumors in type I neurofibromatosis: a pathologic and molecular study. Mod Pathol 2005;18:47584.

5. Corless CL, Fletcher JA, Heinrich MC. Biology of gastrointestinal stromal tumors. J Clin Oncol 2004;22:3813-25.

6. Reiter MJ, Schwope RB, Bui-Mansfield LT, Lisanti CJ, Glasgow SC. Surgical management of retrorectal lesions: what the radiologist needs to know. AJR Am J Roentgenol 2015;204:386-95.
7. Kantarjian HM, Wolff RA, Koller CA. The MD Anderson manual of medical oncology. 2nd ed. New York: McGraw-Hill; 2011.

8. Pappo AS, Janeway KA. Pediatric gastrointestinal stromal tumors. Hematol Oncol Clin North Am 2009;23:15-34.

9. Rubin BP, Singer S, Tsao C, Duensing A, Lux ML, Ruiz R, et al. KIT activation is a ubiquitous feature of gastrointestinal stromal tumors. Cancer Res 2001;61:8118-21.

10. Riddell RH, Petras RE, Williams GT, Sobin LH. Mesenchymal tumors. In: Rosai J, Sobin LH, editors. Tumors of the intestine, atlas of tumor pathology. Washington, DC: Armed Forces Institute Pathology; 2002. p. 325-94.

11. Tzen CY, Mau BL. Analysis of CD117-negative gastrointestinal stromal tumors. World J Gastroenterol 2005;11:1052-5.

12. Mendenhall WM, Zlotecki RA, Scarborough MT, Gibbs CP, Mendenhall NP. Giant cell tumor of bone. Am J Clin Oncol 2006;29: 96-9.

13. Maddah G, Abdollahi A, Etemadrezaie H, Ganjeifar B, Gohari B, Abdollahi $\mathrm{M}$, et al. Problems in diagnosis and treatment of retrorectal tumors: our experience in 50 patients. Acta Med Iran 2016; 54:644-50.

14. Baqué P, Karimdjee B, Iannelli A, Benizri E, Rahili A, Benchimol $D$, et al. Anatomy of the presacral venous plexus: implications for rectal surgery. Surg Radiol Anat 2004;26:355-8.

15. D’Ambra L, Berti S, Bonfante P, Bianchi C, Gianquinto D, Falco E. Hemostatic step-by-step procedure to control presacral bleeding during laparoscopic total mesorectal excision. World J Surg 2009; 33:812-5. 